\title{
PERMANENCIA ESTUDIANTIL EN EDUCACIÓN SUPERIOR: LA VISIÓN DEL DOCENTE
}

\author{
AUTORES: Aída Milena Murillo Zabala ${ }^{1}$ \\ Pedro Jurado de los Santos ${ }^{2}$ \\ DIRECCIÓN PARA CORRESPONDENCIA: (aida.murillo@pi.edu.co)
}

Fecha de recepción: 23/03/2019

Fecha de aceptación: 21/04/2019

\section{RESUMEN}

El presente estudio se centra en la identificación de factores relacionados con la permanencia estudiantil. Fue llevado a cabo en la Institución de Educación Superior Politécnico Internacional (Bogotá, Colombia). Bajo un planteamiento metodológico descriptivo se recolectaron datos con la utilización de cuestionarios ad-hoc y entrevistas (106 y 6 respectivamente). Los resultados evidencian la importancia de las variables calidad y perfil del programa ofertado, en lo relacionado con retención del estudiante. Se observa la influencia de factores motivacionales y de satisfacción personal sobre la persistencia del estudiante dentro del programa. Las variables retención y persistencia son caracterizadas dentro del presente estudio como procesos independientes, susceptibles de estudio, que interactúan e inciden directamente en la permanencia en la Educación Superior.

PALABRAS CLAVE: abandono escolar; retención; Educación Superior; Educación técnica; deserción escolar

\section{STUDENT PERMANENCE IN HIGHER EDUCATION: THE VISION OF THE TEACHER}

\section{ABSTRACT}

This paper is focused on the identification of factors related to students' retention. This study was undertaken at a private Higher Education Institution called Politécnico Internacional (Bogotá, Colombia). To collect the data, some ad-hoc surveys and interviews were utilized (106 and 6 respectively. In relation to the student's retention, results indicate the importance of variables such as quality and profile of the academic program offered. It is also observed

1 Odontóloga. Especialidad en Estomatología Pediátrica. Especialista en Docencia Universitaria. Magister en Educación. Candidata a Doctorado en Educación. Subdirectora académica y de investigación. Politécnico Internacional. Bogotá. Colombia.

${ }^{2}$ Licenciado en Psicología. Licenciado en Ciencias de la Educación. Doctor en Ciencias de la Educación. Profesor Titular de Universidad, Área de Didáctica y Organización Educativa, Facultad de Ciencias de la Educación de la Universidad Autónoma de Barcelona. E-mail:pedro.jurado@uab.cat 
Aída Milena Murillo Zabala, Pedro Jurado de los Santos.

the influence of motivational factors and the personal satisfaction in reference to the students' persistence within the academic program. These two variables, retention and persistence, are characterized in this paper as independent processes, susceptible to study, which interact and have a direct impact on retention in Higher Education.

KEYWORDS: dropout; retention; Higher Education; Technical Education

\section{INTRODUCCIÓN}

El estudio de la permanencia estudiantil en Educación Superior representa una preocupación para las instituciones prestadoras del servicio, las altas tasas de deserción en las universidades representan la oportunidad de mejora en la atención al fenómeno.

Los factores asociados a este fenómeno, responden a una amplia caracterización de variables que inciden directa e indirectamente, sobre la graduación estudiantil.

La persistencia y la retención, hacen parte del estudio de la problemática al tener en cuenta que los factores individuales, sumados a la motivación personal, llevan al estudiante a permanecer en el sistema educativo, esto a la vez soportado por el acompañamiento, guía, y seguimiento que realicen las instituciones para lograr el fin común.

Valorar las prácticas institucionales y las percepciones de los docentes, hace parte de la comprensión requerida para favorecer el entendimiento de las características relacionadas con la permanencia estudiantil en Educación Superior.

El estudio presentado parte de la búsqueda en todo sistema educativo de procurar el éxito y bienestar estudiantil, basado en el presupuesto fundamental: conseguir que los estudiantes que ingresan a las aulas para cursar estudios en cualquier nivel de educación, lo culminen y se gradúen oportunamente.

En este proceso surgen determinados fenómenos objeto de análisis, tales como la deserción, la persistencia y la permanencia estudiantil; las cuales evidencian manifestaciones de que algo en el sistema educativo no responde a ciertas necesidades, llevando a los estudiantes a abandonar sus programas académicos. La deserción en Colombia continúa siendo un signo de alerta para las instituciones de Educación Superior, las estadísticas demuestran que el 46,1\% de los estudiantes del nivel profesional, el 52,1\% del nivel tecnológico, y el 56,9\% del nivel técnico profesional. Ministerio de Educación Nacional (2016), abandonan sus estudios En este trabajo, dejando grandes interrogantes al interior de las instituciones.

Las dudas por el desconocimiento de las razones con relación a la permanencia estudiantil, impulsan a las instituciones a dilucidar los motivos causantes de la deserción, y más importante aún, a estudiar los fenómenos para comprenderlos y entender la dinámica del proceso, creando alternativas de solución que permitan disminuir el porcentaje de estudiantes que abandonan sus estudios.

Por tal motivo el propósito del estudio es: valorar el panorama de la situación vigente de permanencia estudiantil en el nivel de formación técnica profesional del Politécnico Internacional, institución de Educación Superior, desde la postura de los docentes que laboran en la institución.

2 UNESUM-Ciencias. Publicación cuatrimestral. Vol. 3, Año 2019, No. 1 (abril) 


\section{DESARROLLO}

\section{MARCO CONCEPTUAL}

\section{Permanencia estudiantil}

Velásquez (citado en Mendoza et al., 2014), define la permanencia estudiantil como "el proceso que vive el estudiante al ingresar, cursar, y culminar su plan de estudio en el tiempo determinado para ello"; de tal manera que diferentes factores conectados entre sí, como los personales, académicos y familiares, juegan un papel determinante para que los estudiantes finalicen la etapa universitaria. Así mismo, el autor relaciona variables adicionales que condicionan la decisión de los estudiantes, tales como el establecimiento de relaciones sociales al interior de la universidad, la historia académica, la situación socioeconómica, la capacidad de adaptación, la resistencia y la tolerancia a la frustración.

A su vez, Meléndez (citado en Mendoza et al., 2014) la define como el lapso de tiempo que el estudiante toma, desde el ingreso hasta obtener su título profesional. Esta definición corta y precisa es relevante para el desarrollo de la presente investigación, al considerar la permanencia estudiantil como la base para inquietarnos por los factores que inciden en la decisión de un estudiante de no llegar a la obtención del título profesional.

Los factores relacionados con la permanencia estudiantil durante el transcurso de la vida universitaria han sido clasificados y estudiados por diversos autores a través de los años, con el objetivo de encontrar respuestas y propender por la perseverancia del estudiante hasta finalizar. Dentro de diversos modelos de análisis, uno de los de mayor influencia ha sido el trabajado por Tinto (citado en Báez et al., 2010) quien en sus planteamientos reconoce que para hablar de persistencia se deben abarcar no solo los antecedentes familiares, personales y académicos de los estudiantes, sino también las condiciones denominadas por él de integración, que se desarrollan entre la institución y los estudiantes.

La integración a la que hace referencia Tinto (citado en Báez, 2010) atañe a las instituciones en cuanto se convierten en la base estructural para el desarrollo de potenciales desde el ingreso hasta la culminación del proceso académico del estudiante. A este respecto, Pineda (citado en Mendoza, 2014) define la permanencia como "las tareas que realiza la universidad mediante el seguimiento y apoyo sistemático del estudiante para favorecer la culminación exitosa de sus estudios".

Con esta definición se aseveran los conceptos de Tinto, involucrando el rol de las universidades como actores fundamentales para propender por el éxito en la permanencia estudiantil y así lograr avanzar hasta clausurar con la obtención del grado.

\section{La relación permanencia- deserción en Educación Superior}

El término permanencia en el presente estudio, está vinculado al contexto actual y a los avances relacionados con el abandono en Educación Superior; como lo afirma el MEN (2015), "la permanencia se entiende como un tema ocasionado por múltiples causas en el que intervienen diferentes actores y que más allá de un asunto económico, tiene también un impacto directo en el proyecto de vida del estudiante y su familia". El sistema educativo es 
Aída Milena Murillo Zabala, Pedro Jurado de los Santos.

altamente relevante, teniendo en cuenta que el estudiante puede abandonar un programa y trasladarse a otro; puede abandonar una institución y cambiarse a otra; y no necesariamente declinar de su formación superior. En tal medida, el término permanencia engloba una cantidad significativa de variables y características, que deben ser revisadas y analizadas por las instituciones y los sistemas de educación con el fin de comprender y mitigar el abandono estudiantil.

Para la comprensión del abandono estudiantil en este punto, tomamos el interesante resumen que sobre las causas del mismo realizaron Cabrera, Bethencourt, Álvarez, Pérez y González 2006, citados en Elías 2008, los cuales las agrupan en seis categorías: Causas psicoeducativas, evolutivas, familiares, económicas, institucionales, sociales:

Abordando bajo la anterior perspectiva las causas del abandono estudiantil, continuamos con la revisión del fenómeno opuesto en el presente contexto, permanencia estudiantil, sobre la cual diferentes autores, han conceptualizado basados en sus saberes y análisis del tema. Para Meléndez (2008), la permanencia es definida como el tiempo que tarda un estudiante en terminar los requisitos académicos para obtener el título como profesional. A su vez, Velásquez (2010, citado por Mendoza, 2014), la considera haciendo referencia al proceso vivido por el estudiante desde el ingreso, el transcurso del programa y su culminación en un tiempo determinado; teniendo en cuenta factores adicionales como la obtención de promedios ponderados adecuados, establecimiento de relaciones sociales, la formación previa del estudiante, su historia académica, su situación socioeconómica, la capacidad de adaptación, la resistencia y la tolerancia a la frustración.

Sociológicamente, en la literatura se describen dos teorías ligadas directamente con la permanencia de los estudiantes en las aulas; estas según Viale, H (2014) son:

1. El modelo de integración del estudiante (Student integration model) por Spady (1971) y Tinto (1975)

2. El modelo de desgaste del estudiante (Student attrition model) por Bean y Metzner (1985).

\section{MARCO METODOLÓGICO}

El presente estudio se centra en el caso del Politécnico Internacional (PI) Bogotá, Colombia, corresponde al estudio de una institución, en donde se valoran evidencias que permiten la triangulación y proposiciones teóricas que guían el análisis de los datos (Stake, 2007; Yin, 2009); pertenece a los denominados como ex post facto, ya que procura investigar las posibles relaciones de causa-efecto (Cohen, Manion, \& Morrison, 2007), comparando las semejanzas y diferencias que existen entre fenómenos, para descubrir los factores que contribuyen a la aparición de situaciones (Rosado, 2006).

El estudio observa la condición de permanencia estudiantil institucional, buscando respuestas plausibles como factores causales desde la mirada de los docentes; es decir, qué factores están asociados a la permanencia. De igual manera, está encaminado a visibilizar las relaciones entre diferentes variables (Rosado, 2006) dentro de la estructura del PI.

\section{UNESUM-Ciencias. Publicación cuatrimestral. Vol. 3, Año 2019, No. 1 (abril)}


Por medio del enfoque mixto (cuali-cuantitativo) se contrastan resultados y se profundizan los hallazgos encontrados de manera rigurosa.

Cuantitativamente, los cuestionarios ad-hoc fueron utilizados para obtener y analizar información pertinente y precisa en la consecución de los objetivos propuestos.

El cuestionario se aplicó de esta manera, con el fin de recopilar información útil, teniendo en cuenta que este tipo de instrumento cuenta con altos niveles de fidelidad (Del Rincón, 1995).

Como estudio de caso, adicionalmente se busca información de la situación actual de permanencia en la institución, recopilando experiencias, condiciones del contexto y características específicas, Rosado (2006); con el fin de reunir información institucional en datos numéricos, útiles para el análisis de las secuencias e interrelaciones de los factores asociados con la permanencia estudiantil.

Cualitativamente, se pretende entender la realidad de los docentes utilizando instrumentos tales como entrevistas semiestructuradas con el fin de obtener una visión amplia desde los diferentes perfiles y puntos de vista individual. (Bonilla y Rodríguez, 2005, p.160);

\section{Población y muestra}

Cuestionarios ad hoc, diligenciados por los docentes: $107 / 120=89,1 \%$

Entrevistas semiestructuradas: a seis docentes de la institución.

\section{Procedimiento}

Los cuestionarios validados en su versión final, se transcribieron utilizando la herramienta para encuestas Google Drive. Finalizadas las semanas para el diligenciamiento del cuestionario, se consolidó la información y se obtuvieron los datos definitivos mediante el programa SPSS, con los que nos permitimos realizar el análisis descriptivo e inferencial, dando cumplimiento a los objetivos específicos del estudio. De igual manera, las entrevistas fueron transcritas y procesadas para la obtención de la información, mediante el programa AtlasTi.

\section{DISCUSIÓN Y RESULTADOS}

\section{Cuantitativos}

Análisis variables docentes

\section{Edad}

En la muestra de docentes encuestados, el mayor porcentaje de participación, corresponde a los profesores con edades comprendidas entre los 41 y 45 años (39\%), seguidos por los de edades entre 31 y 40 años (20\%). Los docentes con edades entre 25 y 30 años, representan el menor porcentaje de la muestra (8\%), antecedidos por los menores de 25 años con un $15 \%$ de participación en la muestra. 
Aída Milena Murillo Zabala, Pedro Jurado de los Santos.

Tabla 1. Edad de los docentes participantes

\begin{tabular}{llr|r|} 
& & Frecuencia & Porcentaje \\
\hline Edad & & 9 & \\
\cline { 2 - 4 } & De 25 a 30 & 21 & 19,4 \\
\hline & De 31 a 40 & 19 & 17,7 \\
\hline De 41 a 45 & 19 & 17,7 \\
\hline Mayor de 45 & 16 & 14,9 \\
\hline Menor de 25 & Total & 107 & 100,0 \\
\hline
\end{tabular}

Fuente: elaboración propia

\section{Género}

La encuesta fue respondida por 44 mujeres $(41,1 \%)$ y 63 hombres $(58,9 \%)$

\section{Nivel educativo de los docentes}

El nivel educativo de los docentes participantes en el estudio, es en su mayor porcentaje de especialización (38,3\%), seguido por los profesionales en un $(34,5 \%)$. En bajos porcentajes se encuentran los profesores con nivel de educación técnica $(12,1 \%)$ y los que han cursado maestrías $(11,2 \%)$. El porcentaje más bajo corresponde a los docentes que han cursado programas tecnológicos $(3,7 \%)$

\section{Nivel de exigencia del PI}

Es una característica relevante para el estudio de la retención. Por tal motivo, las respuestas de los docentes en los cuestionarios, revelan que la mayoría considera que la exigencia institucional es alta en un 67,3\%. Sin embargo, es importante notar que el $21,5 \%$ de los profesores encuestados, consideran que el nivel de exigencia del PI es muy bajo.

\section{Calidad docente}

Los profesores encuestados consideran contar con buena calidad en docencia (48,6\%), seguidos por los que consideran que la institución cuenta con excelente calidad docente (45,8\%), sólo un 5,6\% considera que las cualidades son suficientes, para desarrollar sus labores dentro del aula.

\section{Recursos que brinda la institución para el desarrollo de las clases}

Son utilizados muy a menudo por los docentes en un $69,1 \%$ el computador, y los medios audiovisuales en un $62,62 \%$. Los que refieren utilizar a menudo son las guías didácticas $(58,8 \%)$, y nunca, las salidas pedagógicas $(42,99 \%)$.

\section{UNESUM-Ciencias. Publicación cuatrimestral. Vol. 3, Año 2019, No. 1 (abril)}




\section{Estrategias pedagógicas}

Para dictar las clases frecuentemente los docentes utilizan las demostraciones activas en un $65,4 \%$, el análisis de casos en un 62,6\%, las simulaciones un 51,4\%, y el ABP en un 46,7\%.

En cuanto a las estrategias que nunca incluyen los docentes para el desarrollo de sus clases, se encuentran las visitas a centros profesionales en un 37,3\%, y la realización de seminarios en un $28,9 \%$.

\section{Recursos ofrecidos a los estudiantes en el PI}

Son considerados por los docentes de excelente accesibilidad, en el caso del auditorio (50,4\%), y las cocinas (46,7\%); mientras que los considerados de regular accesibilidad, son los medios audiovisuales en un 17,7\%, seguidos por la biblioteca en un $14,0 \%$.

En cuanto a adecuación, los recursos ofrecidos a los estudiantes por la institución, en su mayoría son considerados como excelentes por los profesores, siendo el de más alta calificación el auditorio con un 50,4\%, seguido por las cocinas en un $41,1 \%$.

Los calificados como regulares, son nuevamente los medios audiovisuales $(16,8 \%)$ y la biblioteca $(14,9 \%)$.

En cuanto a funcionalidad, los profesores encuestados opinan que los recursos ofrecidos a los estudiantes son excelentes al referirse nuevamente al auditorio $(46.7 \%)$ y a las cocinas $(41,1 \%)$. Los considerados regulares son los medios audiovisuales $(18,6 \%)$, la biblioteca $(14,9 \%)$ y los laboratorios $(14,9 \%)$.

\section{Apoyo financiero}

Otra característica muy pertinente asociada a la retención es el apoyo financiero ofrecido a los estudiantes por la institución. Al respecto, los docentes están totalmente de acuerdo en un 41,1\%, y de acuerdo en un 56,1\%, en que la institución apoya a sus estudiantes en este sentido.

\section{Razones de los estudiantes para abandonar la institución}

Los profesores encuestados consideran como razones muy relevantes para que sus estudiantes tomen la decisión de abandonar la institución son las facilidades de pago 38,3\% y los horarios de estudio en un 19.6\%. Por otro lado, consideran que las razones nada relevantes, para tomar la decisión de abandonar los estudios, son las flexibilidades en los horarios en un 33.64\%, y los servicios de bienestar universitario en un 31,78\%.

\section{Motivación de los estudiantes para asistir al Politécnico}

En la tabla 2 pueden observarse los niveles de significancia para características relacionadas con persistencia estudiantil, en cuanto a factores de motivación para asistir al Politécnico. Siendo altamente significativos el futuro e interés laboral, el compromiso, el aprendizaje, la calidad de vida, del programa, los conocimientos adquiridos, la formación profesional y la preparación para el futuro laboral con un ,000 de significancia; representando diferencias significativas entre los grupos. Seguidos por la metodología docente y el apoyo académico brindado en la institución con un ,003. 
Tabla 2. ANOVA. Motivación asistencia de los estudiantes al P.I

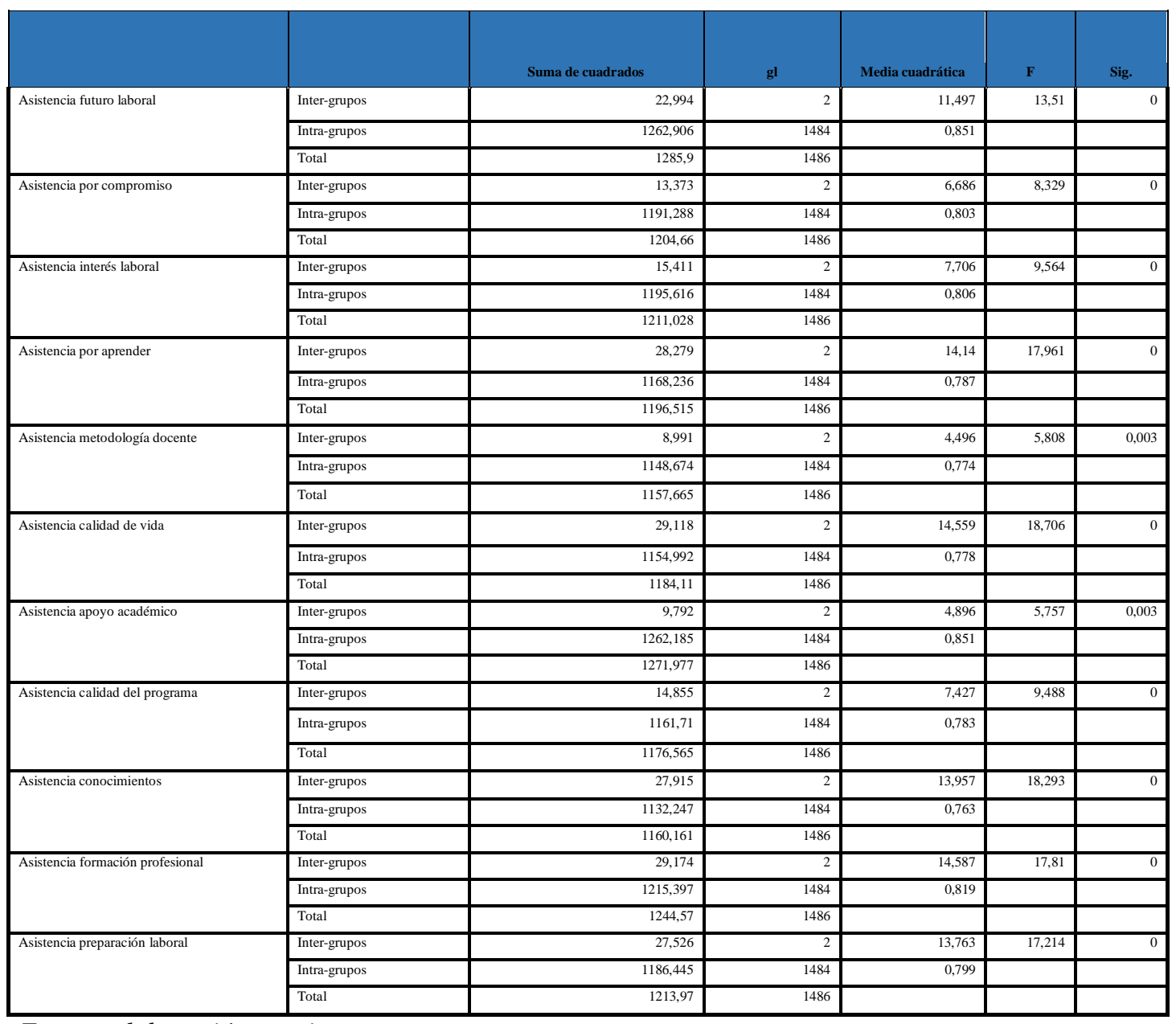

Fuente: elaboración propia

\section{Cualitativos}

La entrevista fue respondida por seis personas que hacen parte de la planta docente del PI: docente titular de gastronomía, docente titular de salud, docente de enfermería, docente de emprendimiento, docente de ingenierías y docente de asignaturas transversales.

\section{Tabla 3. Codificación docentes}

\begin{tabular}{|l|l|l|}
\hline \multicolumn{1}{|c|}{ Código } & \multicolumn{1}{|c|}{ Caracterización } & \multicolumn{1}{c|}{ Número de citas } \\
\hline DcntHosp & Docente Facultad de Hospitalidad & 6 \\
\hline DcntEnf & Docente Facultad de Enfermería & 7 \\
\hline DcntEmp & Docente Facultad de Emprendimiento & 9 \\
\hline DcntIng & Docente Facultad Técnicas de Ingenierías & 4 \\
\hline DcntS & Docente Facultad Salud & 5 \\
\hline DcntT & Docente Área Transversales & 4 \\
\hline
\end{tabular}

8 UNESUM-Ciencias. Publicación cuatrimestral. Vol. 3, Año 2019, No. 1 (abril) 


\section{Fuente: elaboración propia}

Las entrevistas fueron organizadas configurando las preguntas como ejes de análisis. Se procedió a hacer una codificación temática del contenido de las respuestas dadas a cada pregunta, y de esta manera se identificaron categorías emergentes que permitieron la identificación y comprensión de lo planteado por los docentes frente a las preguntas.

Las categorías emergentes fueron relacionadas por tema y se crearon redes visuales que representan los vínculos entre pregunta (centro) y las categorías emergentes (alrededor). En algunos casos, no se realizaron redes debido a que la información identificada no lo permitió.

\section{Factores relacionados con la variable permanencia}

Las instalaciones de la institución, el bienestar universitario, el apoyo académico, administrtivo, entre otros, contribuyen a la permanencia estudiantil en cada institución.

En relación con las instalaciones y el bienestar universitario al interior de la institución, como favorecedores de la interacción social, aspecto fundamental para la permanencia, se identificaron dos tipos de respuestas otorgadas por los docentes, cada una con distintos matices. Por un lado, cuatro docentes manifiestan conformidad con relación a las instalaciones y el bienestar. Por otro lado, dos docentes manifestaron pocos espacios adecuados para interactuar. Gráfica 1

Gráfica 1. Bienestar

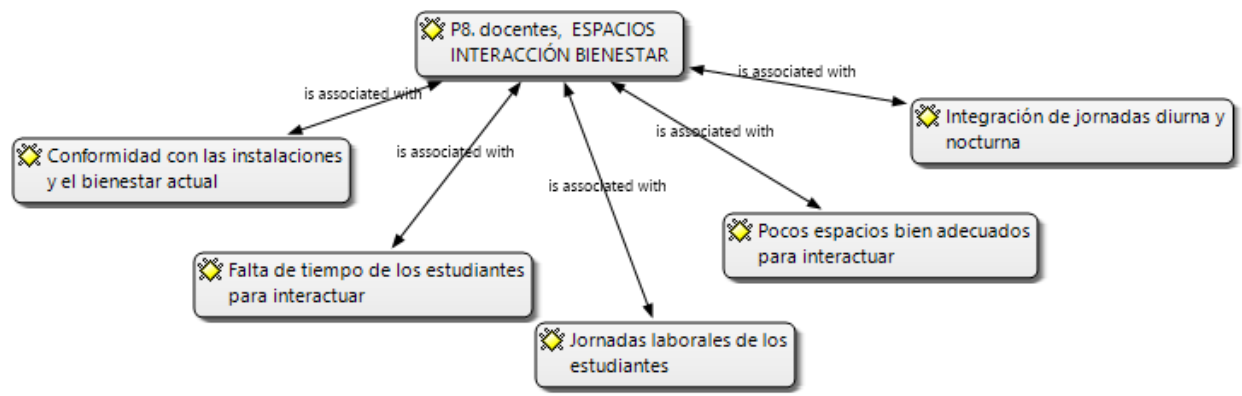

Fuente: elaboración propia

Las jornadas laborales de los estudiantes tienen implicaciones sobre la percepción por parte de los docentes en cuanto a las necesidades de bienestar universitario e interacción social. A esta perspectiva, se suma: "los espacios no son muchos pero están bien adecuados para que la interacción social pueda darse. Además ellos están poco tiempo en la institución, porque vienen y estudian y se van a trabajar". (DcntT). Esto permite concluir que, desde esta posición, la falta de tiempo de los estudiantes hace que no sea necesario mejorar en espacios o infraestructura para la interacción social, y que los espacios que actualmente poseen son suficientes. 
Aprovechando la experiencia de los docentes en la institución, procedemos con el análisis de las razones asociadas desde su percepción, con el abandono de los estudiantes en el aula.

\section{Gráfica 2. Factores abandono de estudios}

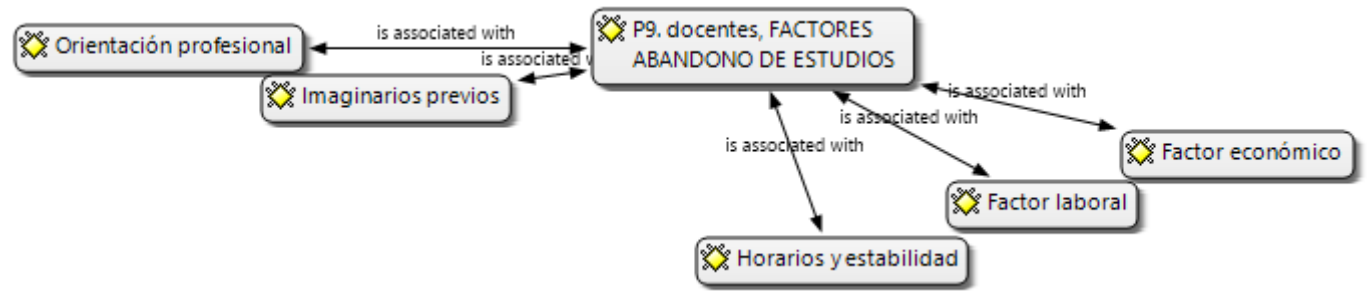

Fuente: elaboración propia

Se evidenció que el factor económico es el factor más relevante en el abandono de los estudios por parte de los estudiantes. Todos los docentes se refirieron a éste, ya sea porque a los estudiantes no les alcanza el dinero para pagar el período académico o los materiales, o porque deben responder por su familia y el dinero no es suficiente para el transporte, el arriendo, la alimentación y los gastos de estudio.

Otro factor (relacionado con el anterior), es el factor laboral vinculado con los horarios y la estabilidad. Aunque a algunos estudiantes les alcance el dinero para estudiar, en ocasiones los horarios laborales no son compatibles con los horarios de estudio, y ellos, en definitiva, no pueden dejar de trabajar.

Otros dos factores que surgieron y están relacionados entre sí, son la orientación profesional y los imaginarios alrededor del estudio y el trabajo. Esto se explica porque por ejemplo "hay muchos que entran sin conocer que es lo que van a estudiar y se retiran" (DcntS). Otros en cambio, creen que estudiar sólo un ciclo les alcanza para obtener suficientes conocimientos dentro del área y para la vida laboral.

Conociendo los factores anteriormente relacionados, se identificaron posiciones encontradas al indagar acerca de los motivos por los cuales los estudiantes abandonarían sus estudios, teniendo en cuenta las consideraciones de los docentes acerca de si ciertas actitudes o comportamientos propios, incidirían en la toma de decisión de un estudiante para no desertar.

10 UNESUM-Ciencias. Publicación cuatrimestral. Vol. 3, Año 2019, No. 1 (abril) 
Gráfica 3. Incidencia de labor docente en la deserción

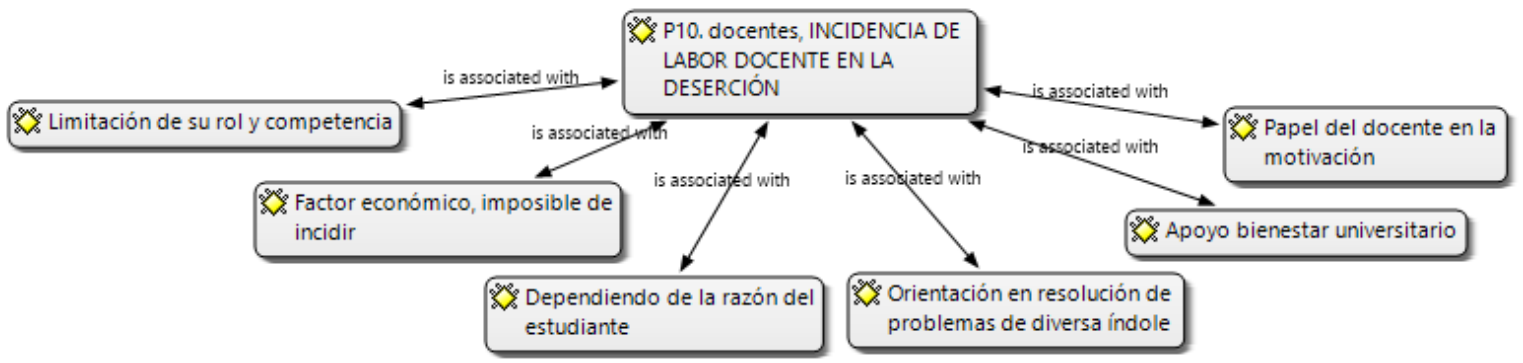

Fuente: elaboración propia

Algunas respuestas manifestaron que la labor del docente incide efectivamente en la toma de decisión de un estudiante frente a la posibilidad de desertar, y otras, que no o que no depende necesariamente de ellos.

En efecto, la opinión manifestada por cuatro docentes, está relacionada con la dependencia de la razón del estudiante de desertar, como factor fundamental de la capacidad de apoyo de los docentes.

Esto, debido a varios aspectos. Por ejemplo, frente al factor económico: “Por los motivos económicos es difícil. Yo les doy apoyo en las clases y oportunidades, pero cuando es por dinero si es muy difícil, ellos definitivamente no pueden y se van." (DcntEmp)

Lo anterior, directamente relacionado con las limitaciones que presentan relacionadas con su rol y competencia como docentes:

Pues yo los acompaño y los guio hasta donde mi competencia y rol docente lo permite. Les trato de motivar y mostrar las cosas buenas que tiene estudiar acá en la institución. Pero si hay algunos que verdaderamente por plata no pueden seguir adelante, y otros también, que no sé a qué vienen, porque en realidad no es mucho lo que les interesa lo que estudian. (DcntHosp).

En contraste, se manifestaron opiniones en las que los docentes hacen lo posible para incidir en que el estudiante no deserte. Para el docente de ingeniería y de transversales, una función del docente es acompañar y estar pendiente de sus estudiantes, aportando continuamente desde la motivación al estudiante, para que este no tenga deseos de abandonar los estudios. "Pienso que sería bueno que todos los docentes nos encargáramos de hacer clases muy motivadoras que le permitan al estudiante mantenerse hasta finalizar la carrera. ”(DcntIng).

\section{CONCLUSIONES}

La permanencia estudiantil en Educación Superior, debe llegar a ser el sistema de prevención que impulse a las Instituciones de Educación Superior a implementar mecanismos y herramientas útiles, de acuerdo al contexto 
Aída Milena Murillo Zabala, Pedro Jurado de los Santos.

específico en que se desenvuelven, a acompañar a los estudiantes desde el ingreso hasta el final, con el objetivo de culminar exitosamente el camino emprendido.

Los espacios abiertos al interior de las instituciones, que promueven la discusión y generación de herramientas y recursos, y sobre todo la innovación y adaptación a los nuevos modelos de educación, reducen la deserción estudiantil, siendo esta el resultado de sistemas de permanencia débiles o poco desarrollados al interior de las universidades.

Teniendo en cuenta la variedad y la problemática de los factores involucrados en la permanencia estudiantil, el modelo de la investigación disgregó las dimensiones relacionadas con el fenómeno, para abordarlo desde la visión de los docentes, buscando facilitar el planteamiento de alternativas útiles para la generación de condiciones que garanticen el objetivo final del estudiante.

Los docentes consideran fuera de los factores económicos y laborales, dos situaciones muy relevantes para la permanencia estudiantil: la orientación vocacional, y los imaginarios que traen los estudiantes al ingresar a la Educación Superior.

La labor del docente incide efectivamente en la toma de decisión de un estudiante frente a la posibilidad de desertar.

De igual manera las áreas administrativas apoyan la satisfacción de los estudiantes en la institución, ofreciendo valores agregados para permanecer en la misma.

Los docentes manifestaron satisfacción y apoyo académico institucional en cuanto a acompañamiento pedagógico, sin embargo en cuanto a congresos y eventos externos consideran que podría ser mayor el apoyo institucional para mantenerse actualizados y relacionados con el sector productivo.

Con el propósito firme de lograr la permanencia estudiantil en las instituciones de Educación Superior, es indispensable disgregar las variables que inciden en el fenómeno, desplegando estrategias para cada una, encontrando resultados pertinentes según la realidad y el contexto institucional. Las variables en mención, están enmarcadas entre las dimensiones retención y persistencia, que sumadas, aportan nociones indispensables para aumentar la permanencia, y disminuir la deserción.

Finalmente, se insiste en la importancia de la creación e implementación, a nivel institucional, de estrategias de evaluación del impacto de los planes en pro de la retención, con el fin de garantizar la efectividad de los procesos y afianzar la estructura interna del proceso generado, para fomentar la permanencia estudiantil.

\section{REFERENCIAS BIBLIOGRÁFICAS}

Báez, C. Rojas, R. Tobar, N. Quintero, J. (2010). Persistencia y graduación: hacia un modelo de retención estudiantil para las instituciones de educación superior en Colombia. Universidad de la Sabana. Facultad de Educación.

Bonilla, E. Rodríguez, P (2005). Más allá del dilema de los métodos. La investigación en ciencias sociales. Bogotá: Editorial. Norma 159-180.

Cohen, L. Manion, L. and Morrison, K. (2007). Research Methods in Education. Sixth ed. New York: Routledge

12 UNESUM-Ciencias. Publicación cuatrimestral. Vol. 3, Año 2019, No. 1 (abril) 
Del Rincón, D. Arnal, J. Latorre, A. Y Sanz, A. (1995). Técnicas de investigación en ciencias sociales. Madrid: Dykinson. Disponible en: http://www.tdx.cat/bitstream/handle/10803/1330/

Elias, M. (2008). Los abandonos universitarios: retos ante el espacio Europeo de educación superior. Estudios sobre educación 15 ESE IO2.

Meléndez, R. Meriño, D. (2008). Estudio sobre deserción y Permanencia académica en la Facultad de Ingeniería de la Universidad de la Guajira desde el II PA 2005 haste el II PA 2007. Universidad de la Guajira. Colombia.

Mendoza, L. Mendoza, U. \& Romero, D. (2014). Permanencia académica: una preocupación de las instituciones de educación superior. Escenarios, 12(2), 130-137.

Ministerio de Educación Nacional, MEN (2009). Deserción estudiantil en la educación superior colombiana. Metodología de seguimiento, diagnóstico y elementos para su prevención. República de Colombia. Disponible en www.mineducacion.gov.co

Ministerio de Educación Nacional, MEN (2015). Estrategias para la permanencia en educación superior: experiencias significativas. República de Colombia. Disponible en www.mineducacion.gov.co

Ministerio de Educación Nacional, MEN (2016). Documento de lineamientos de política pública. Sistema Nacional de Educación Terciaria (SNET): Camino para la inclusión, la equidad y el reconocimiento. República de Colombia. Disponible en www.mineducacion.gov.co

Rosado, M (2006). Metodología de investigación y evaluación. Editorial Trillas.

Stake, R (2007). Investigación con estudio de casos. Madrid: Edcs. Morata. Cuarta edición. Morata. Madrid, España.

Viale, H. (2014). Una aproximación teórica a la deserción estudiantil universitaria. Revista Digital de Investigación en Docencia Universitaria. Año 8. Número 1.

Yin, R. (2009). Case study research. Design and methods. Sage publications. Thousand oaks. 4th 
Aída Milena Murillo Zabala, Pedro Jurado de los Santos.

14 UNESUM-Ciencias. Publicación cuatrimestral. Vol. 3, Año 2019, No. 1 (abril) 